\title{
Aryne-Annulated Porphyrazines as Synthons for Unsymmetrical Phthalocyanine-Related Macrocycles
}

\author{
Sergei I. Vagin, ${ }^{@}$ Carly E. Anderson, and Bernhard Rieger \\ Dedicated to Professor Michael Hanack on the occasion of his $80^{\text {th }}$ birthday \\ Wacker-Lehrstuhl für Makromolekulare Chemie, Technische Universität München, Lichtenbergstraße 4, 85748 Garching b. \\ München, Germany \\ ${ }^{\circledR}$ Corresponding authorE-mail: vagin@tum.de
}

\begin{abstract}
This work surveys the progress of Prof. M. Hanack and his working group in recent years in the field of phthalocyanine synthesis. Special attention is devoted to an approach towards unsymmetrical phthalocyanines and related compounds via generation of unsymmetrical aryne-annulated macrocycles as reactive intermediates.
\end{abstract}

Keywords: Phthalocyanines, porphyrazines, synthesis, unsymmetric derivatisation.

\section{Introduction}

Professor M. Hanack is one of the leading researchers in the field of phthalocyanines, who has published pioneering works on co-facial stacking and conductivity of these multifunctional $\pi$-conjugated macrocycles and has significantly contributed to the development of their synthetic chemistry. ${ }^{[1]}$ Also of note are his works on conductive polymers and luminescent materials. Additionally, he is an author of numerous works in the areas of physical and synthetic organic chemistry of cyclic and polycyclic hydrocarbon derivatives and vinyl cations. Modern organic chemistry textbooks repeatedly refer to his works. ${ }^{[2]}$

In this respect, $S$. Vagin deeply appreciates the opportunity to have benefitted from the scientific experience of Professor Hanack by working under his supervision.

In this survey we will chronologically overview some aspects of the postdoctoral stay of S. Vagin in the group of Professor Hanack and survey the findings with an emphasis on the preparation and properties of aryne-annulated porphyrazines.

\section{Discussion}

When S. Vagin joined the group of M. Hanack, one of their current research interests was the application of phthalocyanines (Pc's) and their parent compounds in fields of material science such as optical limiting (OL). The main idea was to find structure - properties correlations in this class of materials with respect to optical limiting effects. Since the commonly observed phenomena of phthalocyanine aggregation has been found to negatively impact on the optical limiting performance, ${ }^{[3]}$ one of the directions of investigations concerned synthetic design of highly soluble poorly-aggregating phthalocyanine complexes. In addition these phthalocyanines should exhibit a superior light stability for their application as OL materials. In this respect, different strategies have been investigated.
Bulky axial substitution at the central metal coordination site in the macrocyclic core has been demonstrated to be a very efficient tool to inhibit the aggregation of planar $\pi$-conjugated molecules in solution. ${ }^{[4]}$ This approach, however, does not gain control over the fast photobleaching of the intensely coloured phthalocyanines, which originates to a great extent from their relatively low oxidation potential and is remarkably dependent on the nature of peripheral substituents as well as on the central metal. Therefore structural modification of the macrocyclic core and periphery as a tool for the simultaneous control of aggregation behaviour and photostability was considered as an alternative strategy towards OL applicationsuitable materials.

A series of different phthalocyanines and their analogues ranging from porphyrazines to naphthalo- and subphthalocyanines have been prepared and tested for optical limiting. ${ }^{[5]}$ Several valuable features and criteria were identified besides the role of the central metal. Firstly, the influence of electron acceptor groups at the macrocycle was found to favour the OL properties. ${ }^{[5]}$ This resulted in development of macrocyclic systems bearing meta-trifluoromethylphenyl substituents as solubilising and simultaneously electron-deficient groups, which to some extent stabilised the macrocycles towards bleaching. ${ }^{[6]}$ Secondly, an enhancement of the optical limiting performance by extension of the $\pi$-conjugation at the porphyrazine core has become unambiguously supported by experiments. ${ }^{[7]}$ The latter has inspired a thorough investigation of naphthalocyanine systems and a development of novel naphthalocyanine-based structures including polyfluorinated and polybrominated derivatives. ${ }^{[8]}$ On the other hand, dimeric annulated phthalocyanines for optical limiting have attracted attention since they also feature an extended $\pi$-conjugation system with an advantage of higher "metal content" and higher photobleaching stability compared to naphthalocyanines. ${ }^{[9]}$ Following this idea, we have considered various synthetic strategies towards conjugated bisphthalocyanines, including the direct dimerisation of Pc-macrocycles via [2+2] 

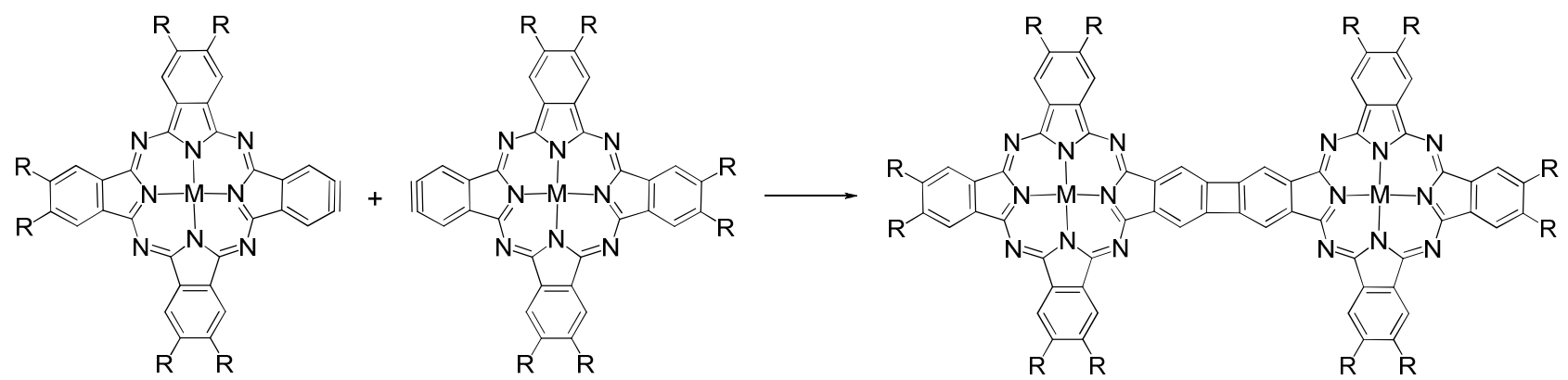

Scheme 1. Hypothetical [2+2] cycloaddition of a dehydrometallophthalocyanine.

cycloaddition as shown in Scheme 1. From the start of the synthesis of dehydrometallophthalocyanine, this project proved to be a major challenge for S. Vagin in Professor Hanack's group.

As can be seen in Scheme 1, the main aim was to generate a dehydrometallophthalocyanine (or aryneannulated porphyrazine) with the idea that this reactive intermediate will undergo the desired dimerisation. In order to avoid an uncontrolled oligomerisation and therefore a numerous and complex product distribution, asymmetric $\mathrm{A}_{3} \mathrm{~B}$-type macrocycles possessing only one aryne moiety needed to be prepared.

Referring to the established chemistry of arynes, ${ }^{[10]}$ we first focused our attention on the generation of aryne-annulated porphyrazine via dehalometallation of a corresponding ortho-dibromo substituted precursor in the presence of a Grignard or similar reagent (see Scheme 2).

The choice of reaction was partially justified by the well-known stability of the dibromo-functionality under the conditions of phthalocyanine synthesis. Additionally, our earlier experiments on arylation of InPc complexes have shown that these macrocycles are relatively stable at least in the presence of excess aryl Grignard reagents. ${ }^{[11]}$ An important requirement was that the precursor should possess sufficient solubility in order to ensure the homogeneity of all subsequent treatments. Since the dibromo-substitution at one of the benzo-moieties of MPc does not favour its solubility, introduction of solubilising groups at the other three benzo-rings of the MPc was necessary. Para-tert-butylphenyl substituents were believed to satisfy this purpose, and the required $\mathrm{A}_{3} \mathrm{~B}$ product was first obtained in a mixture with other statistical products by a template cross-cyclotetramerisation of the two corresponding dinitriles (Scheme 3). Unfortunately, independent of the nature of the central metal and even in the absence of the latter, the mixture could not be satisfactorily separated by column chromatography due to insufficient differences in polarity of the components. An appropriate solution to this problem has been found by apply- ing a well-known method based on a ring expansion reaction of a subphthalocyanine. ${ }^{[12 a]}$ That is, near-complete formation of a zinc complex of $\mathrm{A}_{3} \mathrm{~B}$ phthalocyanine (MPc-1 in Scheme 3 ) is observed upon reaction of $A_{3}$-subphthalocyanine with a corresponding diiminoisoindoline in the presence of zinc acetate, ${ }^{[12 b]}$ as could be seen from MALDI-ToF mass spectral analysis. Interestingly, we could not utilise the solubilsing effect of the meta-trifluoromethylphenyl groups by this approach as they have been shown to undergo partial halogen exchange upon the subphthalocyanine synthesis with boron trichloride. ${ }^{[6 \mathrm{~d}]}$

Parallel to this work, a model ortho-dibromo functionalised $\mathrm{A}_{3} \mathrm{~B}$ porphyrazine system (MPaz-1) was prepared by a cross-condensation of dinitrile precursors as shown in Scheme 4, taking advantage of the facile separation of the corresponding statistical products by column chromatography according to our previous experience. ${ }^{[13]}$

This compound (MPaz-1) was first tested in reactions with metallic magnesium, tert-butylmagnesium chloride and butyl lithium, respectively. It was found that $\mathrm{Mg}$ turnings do not react with the dibromo functionality and $\mathrm{BuLi}$ or ${ }^{t} \mathrm{BuMgCl}$ lead to reductive decomposition of the macrocycle, especially when used in excess, but not to the desired dimeric product. In order to probe the reaction for the intermediate formation of aryne species, these experiments were carried out in the presence of excess furan as a trapping reagent. ${ }^{[13 b]}$ Under such conditions, the reaction with one equivalent of BuLi permitted the detection of some new products along with the starting material. However, on the basis of NMR, UV-Vis spectroscopies, mass spectral analysis and other experiments, they were assigned to metal-free $\mathbf{H}_{2} \mathbf{P a z}-\mathbf{1}$ and related tetraazachlorins. ${ }^{[13 \mathrm{~b}]}$ Obviously, transmetallation in the macrocyclic core takes place before metathesis at the dibromo functionality and the formed $\mathrm{Li}_{2} \mathrm{Pc}$ complex hydrolyses after work up yielding free macrocyclic ligand. The addition of BuLi to the pyrrolic $\mathrm{C}_{\beta}=\mathrm{C}_{\beta}$ double bond of the porphyrazine, resulting in tetraazachlorins, also appears to be faster than metathesis.

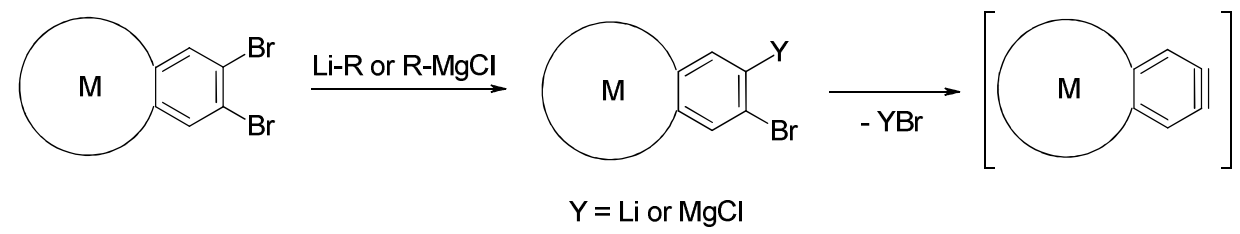

Scheme 2. A proposed pathway towards a dehydrometallophthalocyanine. The circle denotes a Pc-macrocycle. 


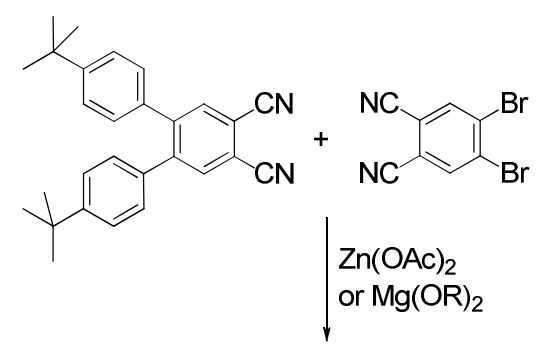

Mixture of statistical products

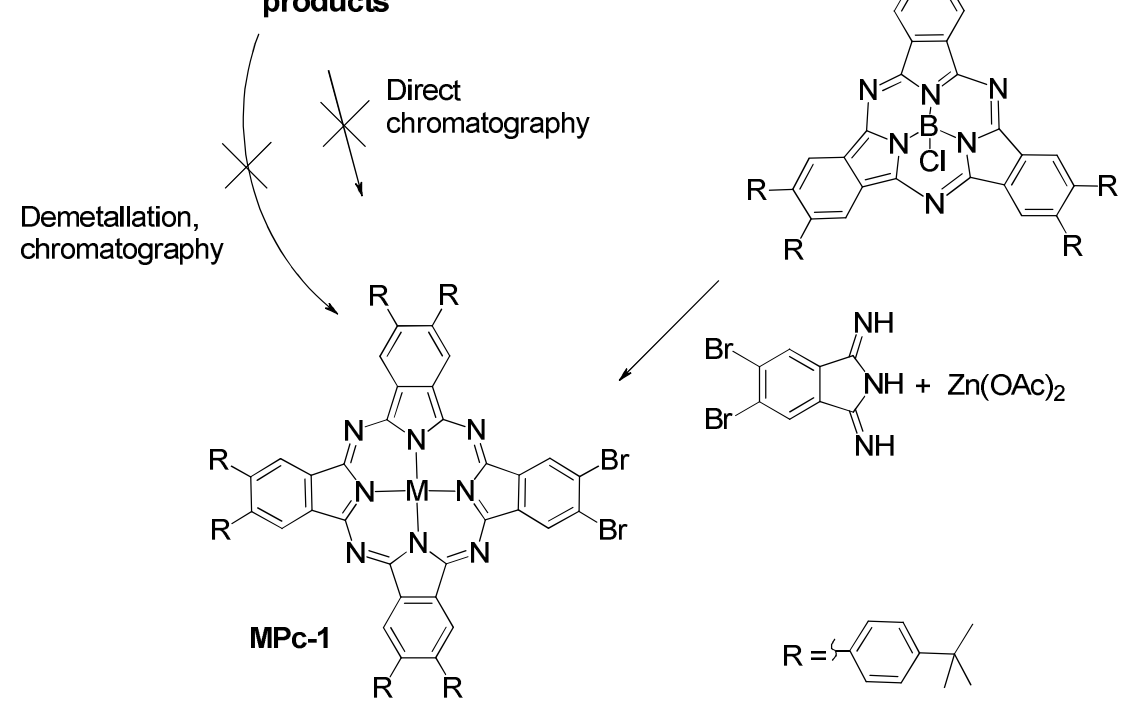

Scheme 3. Synthesis of $\mathrm{A}_{3} \mathrm{~B}$-phthalocyanine with ortho-dibromo functionality.

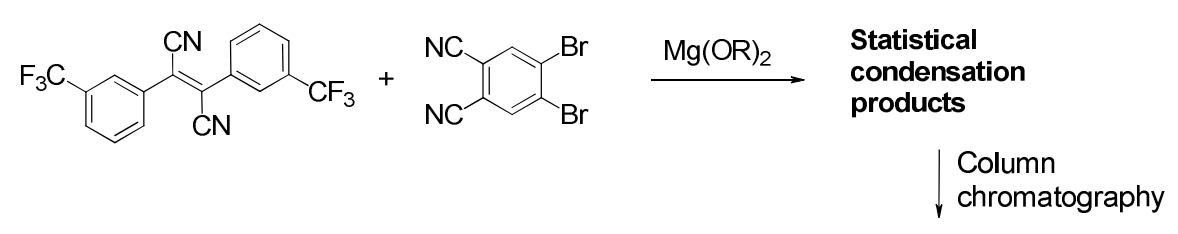

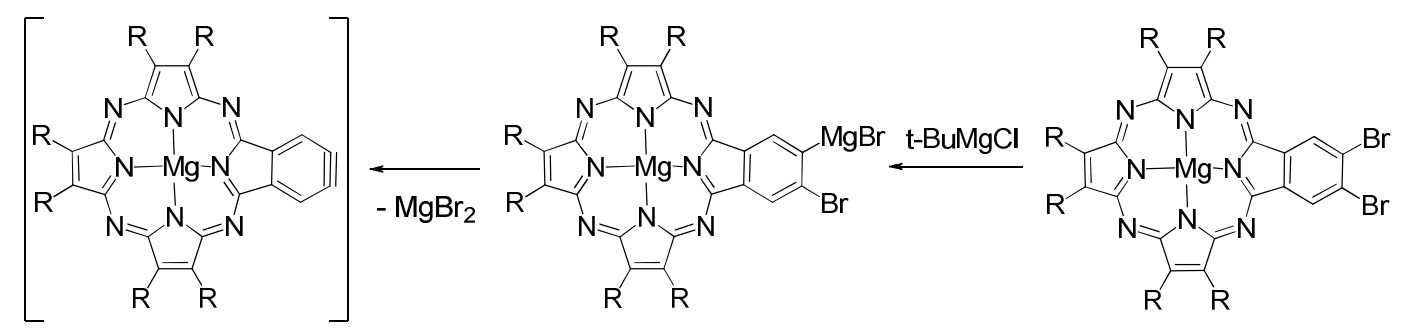
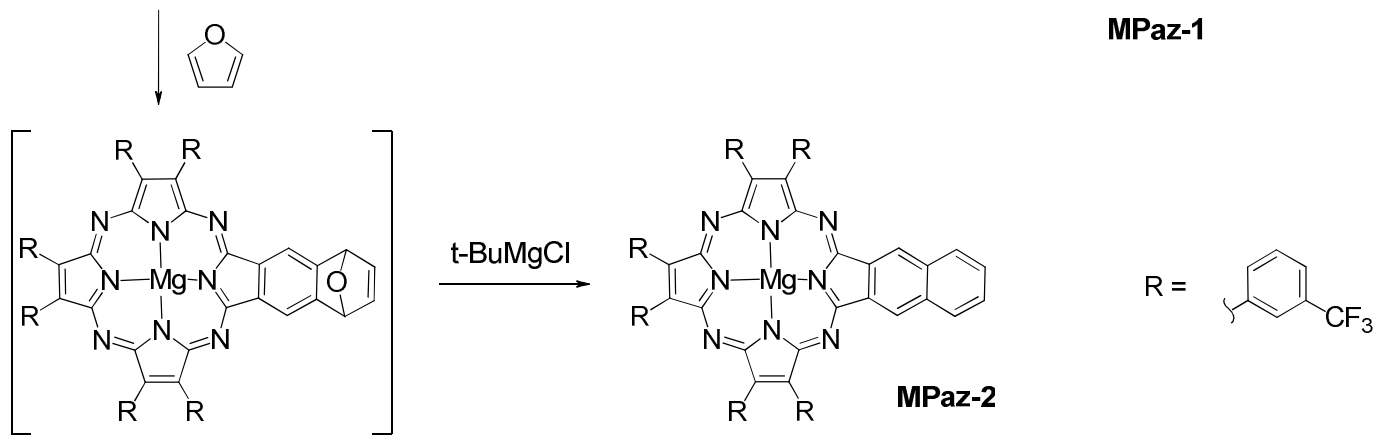

Scheme 4. Synthesis and reactions of mono-(dibromobenzo)porphyrazine.

Finally, in the case of reaction of MPaz-1 with metallic magnesium and ${ }^{t} \mathrm{BuMgCl}$, the formation of the intermediate dehydrobenzoporphyrazine complex was confirmed by isolation of an in-situ aromatised adduct of furan MPaz-2
(Scheme 4), although in a low yield of $24 \%$. The reaction suffered from a high degree of decomposition of the cyclic chromophore, as could be observed by colour change of the reaction mixture from green to brown. Excessively 
long reaction times and higher concentrations of ${ }^{t} \mathrm{BuMgCl}$ were found to promote a more complete decomposition. A side reaction, in which both bromine atoms of the dibromo functionality undergo metathesis to yield the bis-Grignard derivative, was also observed. This has been deduced from the presence of the completely debrominated derivative of MPaz-1 among the products, which could only form after work up of the bis-Grignard compound. Interestingly, when anthracene was applied as an aryne trapping agent, the expected DielsAlder reaction did not occur, but the only isolable product was the debrominated derivative of MPaz-1.

The results were not that encouraging regarding the low yields and propensity towards chromophore destruction under the reaction conditions. Relying on UV-Vis spectroscopic investigations, the decomposition was believed to take place via reduction of the macrocycle followed by its cleavage, which would also explain the irreversibility of the process. This reaction could have been optimised by variation of the solvent, the nature of the Grignard reagent and concentration as well as other factors, but it was not the main goal. Keeping in mind the higher solvolytic stability of phthalocyanine compared to porphyrazine, we assumed that phthalocyanine MPc-1 should show a greater tolerance towards the same conditions.

In contrast, this $\mathrm{A}_{3} \mathrm{~B}$ phthalocyanine with ortho-dibromo functionality underwent rapid decomposition under various conditions upon addition of the Grignard reagent. Neither our goal to prepare a bis-phthalocyanine by direct [2+2] cycloaddition nor generation and trapping of dehydrometallophthalocyanine could be achieved by this method. ${ }^{[12 b]}$

Looking for alternative methods of aryne generation, our attention was drawn to a very elegant and effective procedure which allows preparation of biphenylene via benzyne dimerisation in a very high yield. ${ }^{[14 a]}$ It comprises an oxidative decomposition of $1 N$-aminobenzotriazole functionality in the presence of $\mathrm{Pb}^{\mathrm{IV}}$ salt as shown in Scheme 5.

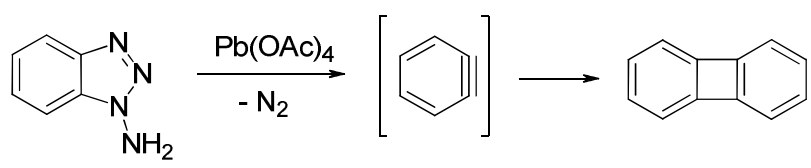

Scheme 5. Generation and dimerisation of benzyne from $1 N$ aminobenzotriazole.

To realise such a strategy with the phthalocyanine system, a $1 N$-aminotriazole moiety at the periphery of the macrocycle was required. Accordingly, an appropriately functionalised phthalonitrile would be necessary for the synthesis of the corresponding Pc. There were no literature reports on such chemistry, and we were anticipating that the $1 \mathrm{~N}$-aminotriazole moiety would not tolerate the conditions of template cross-cyclotetramerisation of dinitriles, whereas its precursor-functionality, namely a triazole moiety, was reported earlier to be stabile under such conditions. ${ }^{[14 b]}$ For this reason, we have developed an improved synthesis of 5,6-dicyano$1 \mathrm{H}$-benzotriazole and have investigated its cross-condensation reactions with other dinitriles. Over the course of our experiments we became familiar with the specifics of these difficult reactions and their frequently individual nature. For example, it was found that the use of alcohols as solvents leads to alkylation of the triazole functionality, excess nickel salt is necessary to protect the triazole ring from decomposition under the conditions of template synthesis, the triazole functionalised macrocycles tend to coordinate excess metal via this triazole moiety upon template synthesis and the target monometallic complexes are hard to isolate. Finally, we could enhance the yield of the desired $\mathrm{A}_{3} \mathrm{~B}$-metallophthalocyanine with an annulated triazole ring up to $32 \%$ (with $42 \%$ theoretically possible because of the statistical factor). ${ }^{[15]}$

Additionally, we have recognised a high synthetic potential of triazole-annulated macrocycles as precursors for unsymmetrically substituted systems which cannot be easily accessed by a template synthesis. For example, the triazole ring undergoes a nearly quantitative $1 N$-sulfonylation with triflic acid anhydride, followed by ring-opening in the presence of strong nucleophiles such as naphtholate leading to systems with an azo-dye-type structural fragment. ${ }^{[16]}$ Alkylation of the triazole ring also proceeds smoothly, but yields a mixture of $1 N$ - and $2 N$ - isomers, which remarkably differ in their UV-Vis absorption properties. ${ }^{[15]}$ We were able to directly aminate the annulated triazole ring by reaction with 2,4-dinitro- $O$-aminophenol (DNAP) in very good yields. ${ }^{[12,17]}$ In this case, the reaction was found to selectively afford $1 N$-amino derivatives (MPc-2 and MPaz-3, respectively, Scheme 6), the formation of which could be easily monitored in situ by UV-Vis spectroscopy. The isomeric purity is unambiguously determined from the NMR spectra of the products.

Here it is worth noting that the choice of substituents in the macrocycles MPc-2 and MPaz-3 was dictated predominantly by the accessibility of their corresponding dinitriles, the solubility effects and finally the relative simplicity of their ${ }^{1} \mathrm{H}$ NMR spectra. Indeed, these peripheral groups seem to optimally satisfy such requirements and are ideal for the preparation of model macrocycles to study the unsymmetrical modification.
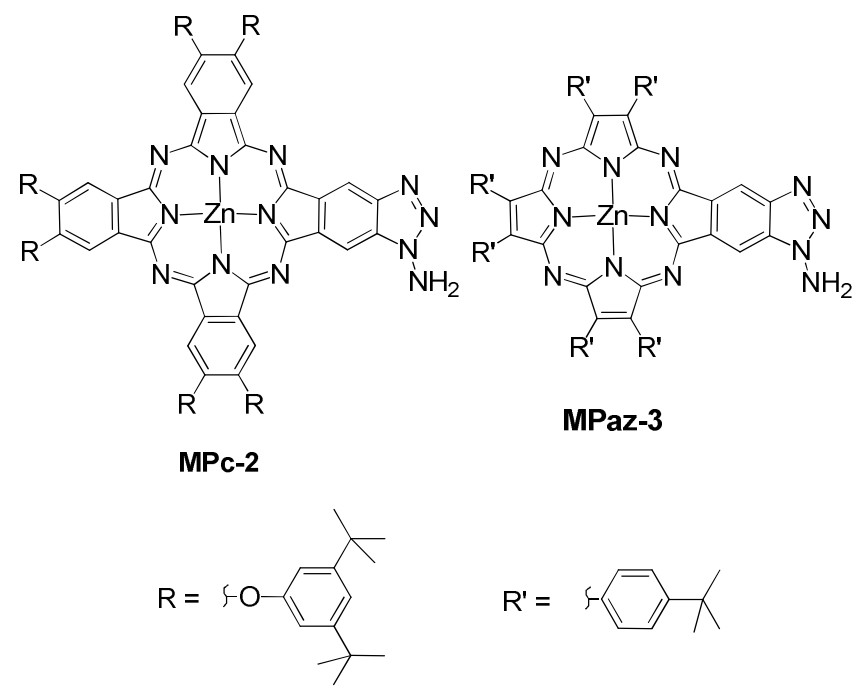

Scheme 6. $1 N$-aminotriazole-annulated macrocyclic complexes.

Our first experiments on generation of aryne-annulated macrocyclic species from the corresponding aminotriazolefunctionalised derivatives were carried out using phthalocyanine MPc-2. In this respect, we first tested the oxidation of MPc-2 by $\mathrm{Pb}(\mathrm{OAc})_{4}$ in the presence of different dienes. It was 


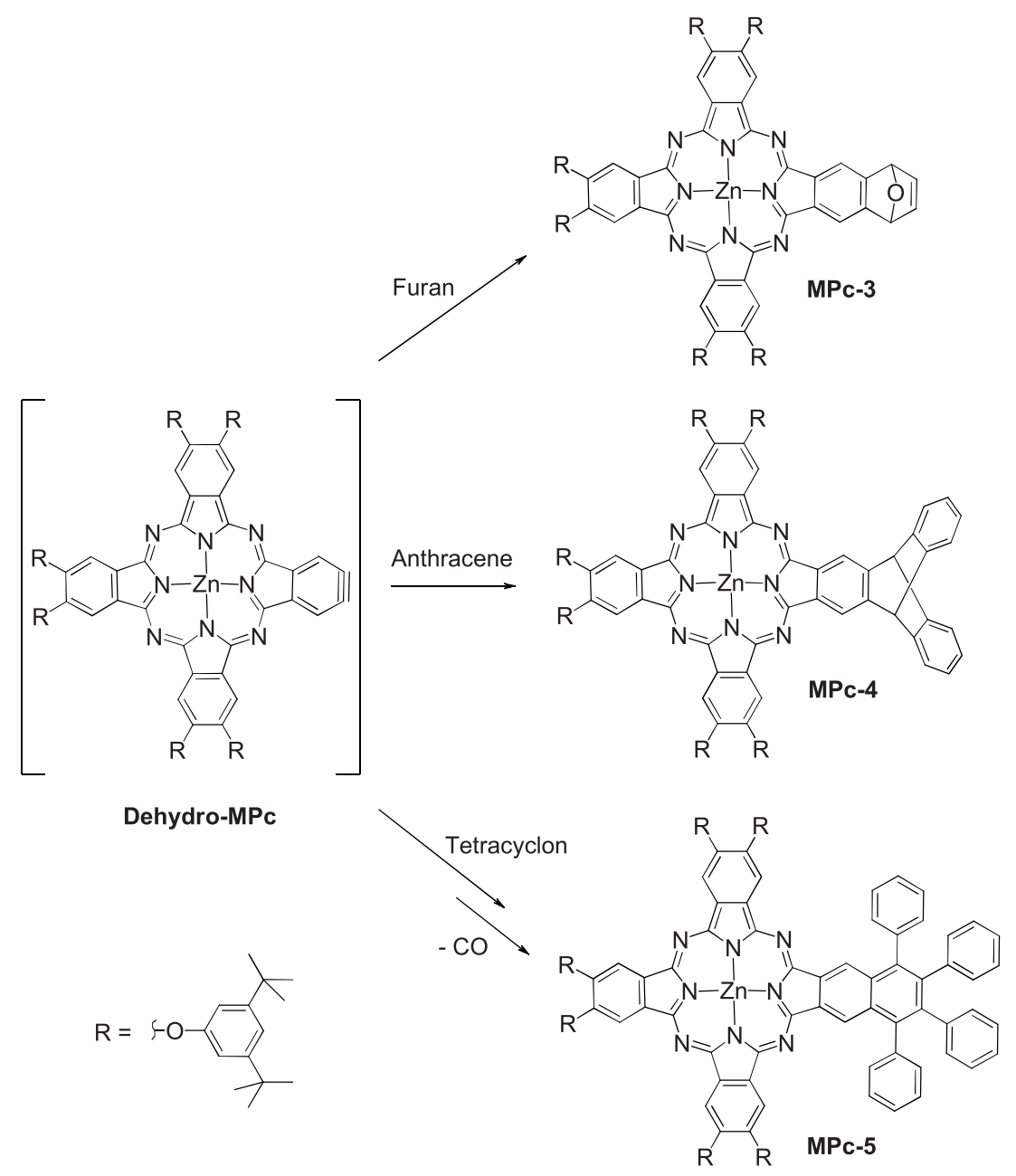

Scheme 7. Reactions of Dehydro-MPc with different dienes.
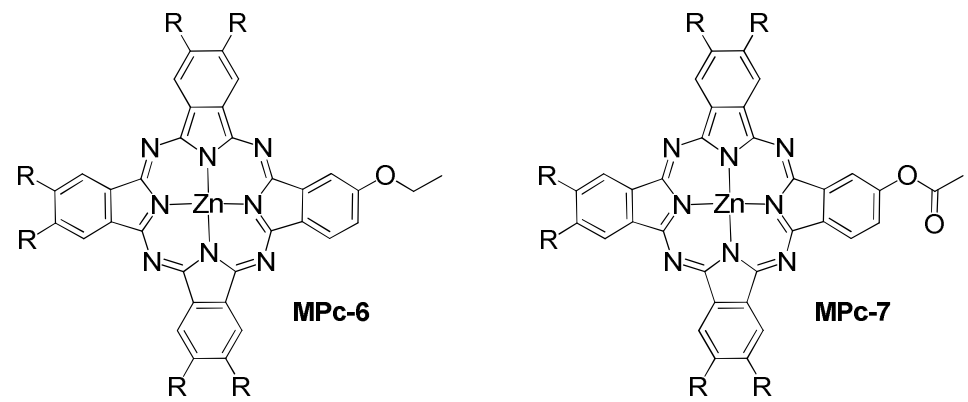<smiles>[R]=[R]Oc1cc(C(C)(C)C)cc(C(C)(C)C)c1</smiles>

Scheme 8. Reaction products of Dehydro-MPc with diethyl ether and with acetic acid.

found that oxidation of both the aminotriazole moiety and Pcmacrocycle can take place upon addition of $\mathrm{Pb}(\mathrm{OAc})_{4}$. The reaction outcome, however, is strongly dependent on the nature of the solvent and can selectively lead to oxidation of the aminotriazole fragment and its subsequent decomposition by elimination of $\mathrm{N}_{2}$ when carried out in diethyl ether with only one equivalent of lead(IV). The generated dehydrophthalocyanine (Dehydro-MPc) reacts rapidly with an added diene to give a corresponding Diels-Alder cycloaddition product. By this approach, we were able to prepare a range of unsymmetrical phthalocyanine complexes shown in Scheme 7. ${ }^{[12 b]}$

Non-coordinating solvents such as chloroform or benzene completely preclude the selectivity of oxidation resulting in bleaching of the solution, and we could not use such solvents for generation of dehydrometallophthalocyanine even in the presence of dienes. Subsequent addition of reducing agents such as $\mathrm{NaBH}_{4}$ or catechols allowed only partial recovery of the chromophore, the majority underwent irreversible decomposition. Strongly coordinating as well as reducing solvents, i.e. pyridine and other amines, deactivate $\mathrm{Pb}(\mathrm{OAc})_{4}$ so that no reaction occurs.

As we were interested in dimerisation of phthalocyanines by [2+2] cycloaddition, our next step was the investigation of dehydrophthalocyanine generation in absence of trapping reagents. We were expecting that $[2+2]$ cycloaddition would take place in diethyl ether upon addition of $\mathrm{Pb}(\mathrm{OAc})_{4}$ to MPc-2. In contrast, no dimeric phthalocyanines but only addition products of diethyl ether and of acetic acid to a triple bond of dehydrometallophthalocyanine were detected under such conditions (MPc-6 and MPc-7 respective- 
ly, see Scheme 8). ${ }^{[17]}$

Since the unsubstituted benzyne does not react with ethers under such conditions but forms biphenylene, the following conclusion is reached: either the reactivity of the triple bond in Dehydro-MPc is much higher and decreases the lifetime of this intermediate by a fast reaction with a slightly nucleophilic solvent molecule, or an increased steric hindrance due to the bulky substituents at the macrocycle periphery prohibits the dimerisation. In order to further examine this situation, experiments were continued with MPaz-3, for which a increased oxidation stability of the macrocycle was expected and, therefore, other solvents could be applied in the oxidation reaction. Indeed, this macrocycle does not undergo oxidative decomposition even in the presence of excess lead(IV) when the reaction is carried out in ethers or even in benzene. ${ }^{[17]}$ However, reaction of MPaz-3 with $\mathrm{Pb}(\mathrm{OAc})_{4}$ in these solvents again resulted in addition of solvent molecules, with even benzene reacting with the triple bond of intermediate benzyne-annulated porphyrazine as a diene, yielding MPaz-4 as the main product (Scheme 9). In THF the oxidation of MPaz-3 proceeded with the formation of more than six addition products (various aryl ether and ester derivatives), but no desired dimer was observed.

Under the same reaction conditions simple $1 N$ aminobenzotriazole always reacted with formation of biphenylene in good yield, and no benzobarellene as an addition product of benzyne to benzene was detected. This again indicated a much higher reactivity of aryne-annulated porphyrazine in comparison with unsubstituted benzyne, so that only non-polar, non-aromatic solvents such as chloroform or dichloromethane should be inert enough to allow the reaction between two aryne-annulated porphyrazine species. Unfortunately, in case of MPaz-3 such solvents were unsuitable for oxidation of the aminotriazole functionality since macrocycle decomposition was predominant.

These experiments do not provide an unambiguous proof of whether a steric hindrance or an increased reactivity of the triple bond in aryne-annulated porphyrazines prohibits the dimerisation. Writing this review, we have realised that an additional test, which probed the oxidation of equimolar amounts of aminotriazole-annulated macrocycle and $1 N$-aminobenzotriazole in benzene would have helped to investigate the role of steric effects. If no cross-cycloaddition product would have been detected, the main factor preventing the dimerisation of the macrocycle has to be its high reactivity. Otherwise, the steric hindrance should be controlling the cycloaddition. Unfortunately, this experiment has not been carried out.

On the other hand, the sterically crowded diene tetraphenylcyclopentadienone (tetracyclon) has been smoothly reacted with MPc-2 in the presence of $\mathrm{Pb}(\mathrm{OAc})_{4}$, and the yield of the corresponding Diels-Alder adduct was comparable with that of the furan adduct. ${ }^{[12 b]}$ Furan cannot be considered as sterically crowded, thus speculatively ruling out a strong steric influence of the peripheral substituents at Dehydro-MPc for this type of reaction. Despite the bulkiness of these substituents, they are conformationally flexible due to the ether linkage with the macrocycle.

In case of Benzyno-MPaz, molecular modelling of

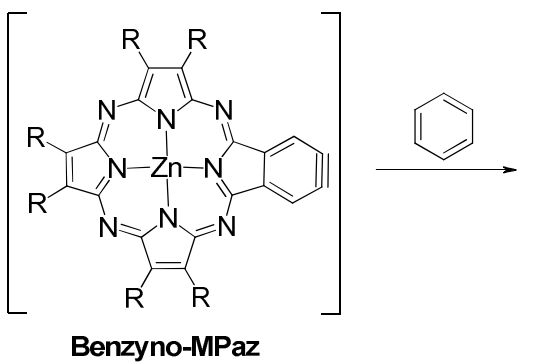

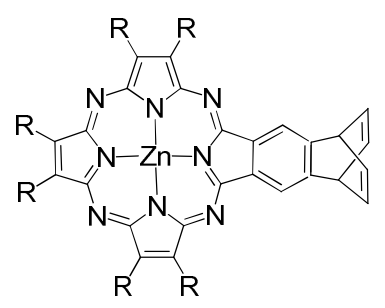

MPaz-4

Scheme 9. Reaction of Benzyno-MPaz with benzene.

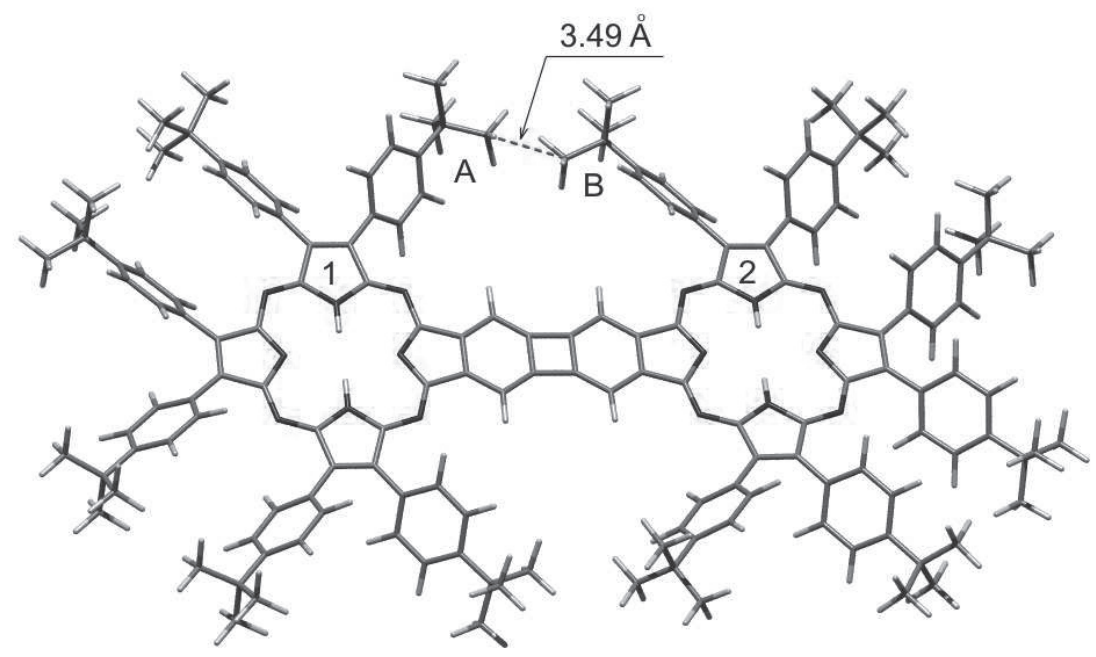

Figure 1. Model structure of a hypothetical dimer of Benzyno- $\mathbf{H}_{2} \mathbf{P a z}$. 
its metal-free analogue using the MM+ method with a subsequent semi-empirical AM1 calculation reveals only negligible steric demands for the corresponding hypothetical $[2+2]$ cycloaddition product. A weak distortion of the planar $\pi$-conjugated system with $c a .4 .2^{\circ}$ angle between the mean planes of the two macrocycles was obtained. The mean planes of pyrrole rings 1 and 2 (Figure 1) form an angle of $9.4^{\circ}$. The smallest distance between the carbon atoms of neighbouring tert-butyl groups $\mathrm{A}$ and $\mathrm{B}$ is found to be $3.5 \AA$, which is close to the sum of van-der-Waals radii. This close contact causes a tilting of the corresponding tert-butylphenyl groups with a torsion angle of $14^{\circ}$ and hinders the rotation of the tert-butyl groups, which may contribute to the stronger change of negative entropy for such dimerisation reactions compared to benzyne. We have also calculated the enthalpy of dimerisation of benzyne to biphenylene as well as of Benzyno- $\mathbf{H}_{2} \mathbf{P a z}$ to the hypothetical dimer as a difference of the calculated heats of formation. The obtained values $(-160 \mathrm{kcal} / \mathrm{mol})$ differ by only $0.5 \mathrm{kcal} / \mathrm{mol}$, with a higher exothermic effect of dimerisation being found for benzyne.

Taking into account the fact that porphyrazineannulated arynes easily cleave ether functionalities in contrast to the unsubstituted benzyne, we have concluded that they feature a higher electrophilicity of the triple bond, which is probably due to the (-)I effect of the macrocycle. The selectivity of benzyne towards addition of acetic acid in diethyl ether solution in contrast to the reaction of DehydroMPc, which shows addition of both acetic acid and diethyl ether, also supports this conclusion. ${ }^{[17]}$ Because of its high electrophilicity, the reaction of such a triple bond can theoretically occur at any electron rich position including the macrocycle itself. For example, pyrrolic fragments could act as a diene fragment (similar to the case of furan or benzene addition), but we could never observe such products in our experiments. Again, either their formation is strongly sterically hindered or the reactions with solvent molecules occur prior to reaction with the macrocycle. The second argument is more probable in our opinion, since we always had to use a high excess of trapping diene in order to achieve a good yield of the cycloaddition product, otherwise products of solvent addition dominated the product mixture.

\section{Conclusion}

In summary, our work on aryne-annulated porphyrazines has shown that these intermediates can be very effectively generated starting from aminotriazole-annulated species. They are very useful synthons for soluble unsymmetrical porphyrazines (phthalocyanines) which would not be accessible by a common template cross-condensation. We have shown that they easily undergo Diels-Alder-type cycloaddition reactions with dienes and even with benzene, as well as addition of weak nucleophiles such as diethyl ether, THF or acetate to the triple bond, to give a range of new unsymmetrically-functionalised macrocyclic products. However, the direct dimerisation of macrocycles via the triple bond could not be achieved, most probably because of the high reactivity of the intermediates as well as due to their steri- cally demanding nature. The role of both factors remains, unfortunately, not clarified experimentally. With this review we hope to reawake an interest in the chemical community of phthalocyanine chemistry concerning the developed approach towards unsymmetric macrocycles as well as to motivate further investigations in this promising field.

\section{References}

1. Hanack M., Dini D. Stacked Polymeric Phthalocyanines: Synthesis and Structure-Related Properties. In: The Porphyrin Handbook (Kadish K.M., Smith K.M., Guilard R., Eds.). Academic Press, 2003, Vol. 18, 251-280.

2. Smith M.B., March J.March's Advanced Organic Chemistry" (5 ${ }^{\text {th }}$ Edition). Ney-York: J. Wiley \& Sons Inc. 2001, 2101 p.

3. Shirk J.S., Pong R.G.S., Flom S.R., Heckmann H., Hanack M. J. Phys. Chem. A 2000, 104, 1438-1449.

4. Hanack M., Dini D., Barthel M., Vagin S. Chem. Record 2002, 2, 129-148.

5. a) O'Flaherty S.M., Hold S.V., Cook M.J., Torres T., Chen Y., Hanack M., Blau W.J. Adv. Mater. 2003, 15, 19-32; b) Dini D., Yang G.Y., Hanack M. J. Chem. Phys. 2003, 119, 4857-4864; c) Dini D., Hanack M., Egelhaaf H.-J., Sancho-Garcia J.C., Cornil J. J. Phys. Chem. B 2005, 109, 5425-5432.

6. a) Vagin S., Barthel M., Dini D., Hanack M. Inorg. Chem. 2003, 42, 2683-2694; b) Vagin S., Hanack M. Eur. J. Org. Chem. 2003, 2661-2669; c) Vagin S., Hanack M. Eur. J. Org. Chem. 2004, 600-606; d) Dini D., Vagin S., Hanack M., Amendola V., Meneghetti M. Chem. Commun. 2005, 3796-3798.

7. a) Nalwa H.S., Hanack M., Powlasky G., Engel M.K. Chem. Phys. 1999, 69, 308-313; b) Vagin S., Yang G.Y., Lee M.K.Y., Hanack M. Optics Commun. 2003, 228, 119-125.

8. a) Yang G.Y., Hanack M., Lee Y.W., Chen Y., Lee M.K.Y., Dini D. Chem. Eur. J. 2003, 9, 2758-2762; b) Yang Y., Hanack M., Lee Y.W., Dini D., Pan J.F. Adv. Mater. 2005, 17, 875- 879; c) Dini D., Calvete M.J.F., Hanack M., Pong R.G.S., Flom S.R., Shirk J.S. J. Phys. Chem. B 2006, 110, 12230-12239; d) Li Y., Dini D., Calvete M.J.F., Hanack M., Sun W. J. Phys. Chem. A 2008, 112, 472-480.

9. a) M. Calvete, M. Hanack, Eur. J. Org. Chem. 2003, 20802083; b) M. J. F. Calvete, D. Dini, S. R. Flom, M. Hanack, R. G. S. Pong, J. S. Shirk, Eur. J. Org. Chem. 2005, 3499-3509; c) M. J. F. Calvete, D. Dini , M. Hanack, J. C. Sancho-Garcia, W. Chen, W. Ji, J. Mol. Modeling 2006, 12, 543-550.

10. Hoffman R.W.Dehydrobenzene and Cycloalkynes (Organic Chemistry. A Series of Monographs, Vol. 11). Academic Press, 1967.

11. Hanack M., Heckmann H. Eur. J. Inorg. Chem. 1998, 367-373.

12. a) Geyer M., Plenzig F., Rauschnabel J., Hanack M., del Rey B., Sastre A., Torres T. Synthesis 1996, 1139-1151; b) Vagin S.I., Frickenschmidt A., Kammerer B., Hanack M. Chem. Eur. J. 2005, 11, 6568-6573.

13. a) Vagin S.I., Hanack M. Eur. J. Org. Chem. 2002, 2859-2865; b) Vagin S., Hanack M., Kammerer B., Frickenschmidt A. Eur. J. Org. Chem. 2004, 4245-4250.

14. a) Campbell C.D., Rees C.W. J. Chem. Soc. C 1969, 742-747; b) Balakirev A.E., Maizlish V.E., Shaposhnikov G.P. Russ. J. Gen. Chem. 2002, 72, 311-314.

15. Vagin S.I., Hanack M. J. Porphyrins Phthalocyanines 2006, 10, 1309-1318.

16. Àlvarez Micó X., Vagin S.I., Subramanian L.R., Ziegler T., Hanack M. Eur. J. Org. Chem. 2005, 4328-4337.

17. Vagin S.I., Frickenschmidt A., Kammerer B., Hanack M. Chem. Eur. J. 2007, 13, 885-991. 